\title{
Wetting and adhesion properties of quasicrystals and complex metallic alloys
}

\author{
Jean-Marie Dubois ${ }^{1 *}$ and Esther Belin-Ferré2
}

*Correspondence: jean-marie.dubois@univlorraine.fr

${ }^{1}$ Institut Jean Lamour (CNRS-Université de Lorraine UMR 7198), Parc de Saurupt, CS54840, 54011 Nancy

Cedex, France

Full list of author information is available at the end of the article

\begin{abstract}
This paper focuses at wetting on Al-based quasicrystals and complex metallic alloys (CMAs), which comprise a significant number of crystalline compounds of changing lattice complexity, according to composition. Such compounds are thermodynamically stable and may be prepared into various sample shapes that allow easy measurement of surface physical properties in air. Surface energy $\left(\gamma_{S}\right)$ is one of the few fundamental properties of condensed matter: it defines the equilibrium shape of a crystal, it determines the interfacial behaviour of any piece of liquid or solid against another body, etc. The paper summarizes our attempts to determine the surface energy of a large variety of CMAs, including the stable, icosahedral AICuFe and AIPdMn quasicrystals, all equipped with their native oxide layer when placed in ambient conditions. Experimental evidence is given that the surface energy correlates to the electronic density of states underneath the oxide layer as long as its thickness remains below $10 \mathrm{~nm}$. Correlation to the thickness of the oxide on the one hand and on the other to specific features of the electronic density of states will be emphasized, in line with the varying complexity of the studied CMA compounds. Potential application to low-stick cookware will be addressed with a view at finding alternatives to fluorinated surface layers.
\end{abstract}

Keywords: Wetting, Quasicrystals, Complex intermetallics, Adhesion, Oxidation

\section{Background}

At thermodynamic equilibrium, the contact angle $\theta$ of a minute droplet of a liquid deposited onto the (supposedly perfectly flat and horizontal) surface of a solid reflects the balance between so-called surface tensions exerted by its own surface (in the presence of its vapour), the surface tension of the solid (also in the presence of the vapour of the liquid), and the interfacial tension between solid and liquid. The equilibrium shape of the droplet is schematized in Fig. 1 (where symbols are defined) and is summarized by the famous Young's equation:

$$
\cos \vartheta=\left(\gamma_{S V}-\gamma_{S L}\right) / \gamma_{L}
$$

An abundant literature is available about this topic [1-5], which is relevant for lots of phenomena at work in nature (e.g. a dewdrop trapped in a spider net), in industry (e.g. oil extraction, textile tint), and of course in every days life (e.g. grilling a steak in a frying pan is very much a matter of adhesion between the hot surface and a piece of matter that contains around $80 \%$ of water). In this article, we study the equilibrium shape of

(C) 2015 Dubois and Belin-Ferré This article is distributed under the terms of the Creative Commons Attribution 4.0 International License (http://creativecommons.org/licenses/by/4.0/), which permits unrestricted use, distribution, and reproduction in any medium, provided you give appropriate credit to the original author(s) and the source, provide a link to the Creative Commons license, and indicate if changes were made. 


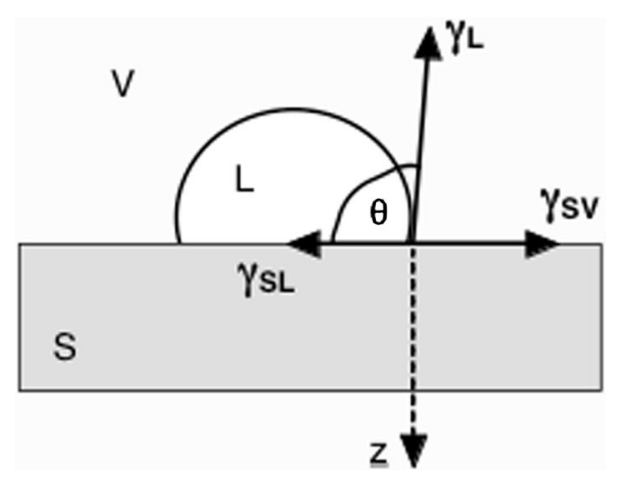

Fig. 1 The equilibrium shape of a minute droplet of a liquid $L$, in the presence of its vapour $V$ and deposited on the perfectly flat, horizontal surface of solid $\mathrm{S}$, reflects the balance between the liquid surface tension $\gamma_{\mathrm{L}}$, the surface tension of the solid in the vapour atmosphere $\gamma_{S V}$, and $\gamma_{S L}$ the interfacial tension between liquid and solid. If roughness effects are negligible, as well as if the difference between $\gamma_{S V}$ and $\gamma_{S L}$ is small, the contact angle $\theta$ is well defined and measurable by optical shape-profiling methods. This well-known figure usually omits the reaction of the solid under the liquid to balance surface tension effects (neglecting gravity). Our article considers this component along the z-direction explicitly

droplets of various liquids put in contact with the surface of intermetallic alloys formed by alloying aluminium with transition metals such as $\mathrm{Cu}, \mathrm{Cr}$ or Fe. Such compounds encompass a broad variety of crystal structures, including quasicrystals first discovered by Shechtman et al. in 1982 in melt spun Al-Mn alloys [6], but also forming stable compounds as was shown few years later by Tsai and his co-workers at Tohoku University, Japan $[7,8]$. In strong contrast to normal metallic alloys and even more so, pure metals like aluminium, the contact angle that we found is large, and is equal to or larger than $90^{\circ}$ although the metallic samples are covered by a thin layer of their native oxide when handled in ambient atmosphere.

The origin of adhesion is usually assigned to extreme surface interactions and is split into two components: one arising from the constant movement of electrostatic charges within the interfacial region (few atomic distances thick) between liquid and solid, or socalled Lifshitz-van der Waals forces on the one hand, and Lewis acid-base interactions on the other hand, which reflect the exchange of charges through the interfacial region [9]. They will be labelled with $\mathrm{LW}$ and $\mathrm{AB}$ subscripts, respectively, in the following. In fact, we will assign the $A B$ subscript to index every kind of interaction (dangling bonds for instance) that is not of LW type. Having this in mind, we can define the reversible adhesion energy $W_{\mathrm{L}}$ of liquid $\mathrm{L}$ on solid $\mathrm{S}$ at thermodynamic equilibrium by the following equation [9]:

$$
W_{L}=\gamma_{S}+\gamma_{L}-\gamma_{S L}
$$

where $\gamma_{\mathrm{i}}, \mathrm{i}=\mathrm{S}$ or $\mathrm{L}$, is the surface energy of material $\mathrm{S}$ or $\mathrm{L}, \gamma_{\mathrm{SL}}$ is the interfacial energy between $\mathrm{S}$ and $\mathrm{L}$, and all $\gamma^{\prime} \mathrm{s}$ are taken identical, respectively, to their surface tension counterparts defined in Fig. 1. Combining Eqs. (1) and (2) leads then to:

$$
W_{L}=\gamma_{L}(1+\cos \vartheta)+\Pi
$$

where $\Pi=\gamma_{\mathrm{S}}-\gamma_{\mathrm{SL}}$ is called the film pressure. This term becomes negligible whenever the contact angle is large enough, which also means that it is well defined, or in other 


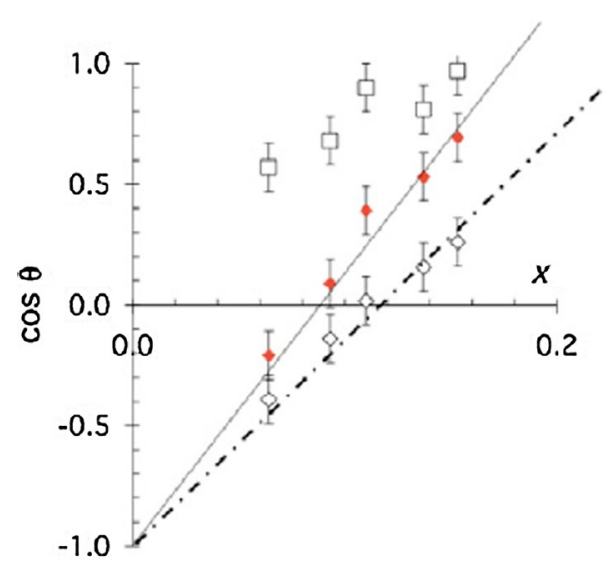

Fig. 2 Plot of $\cos \theta$ versus $x$ (see text) for 5 different liquids (see Table 1) and single crystalline alumina (top curve), single grained icosahedral APM (middle curve) and PTFE (bottom curve). Unlike its covering $\mathrm{Al}_{2} \mathrm{O}_{3}$ oxide (and major component Al, not shown here), the quasicrystal behaves like the apolar PTFE, showing clear evidence that no $A B$ interactions take place at its surface

words, the liquid does not spread out on the solid surface to form a film. We checked that the film pressure is negligible on most of our samples, except pure metals and oxides. Assuming then that $\Pi \rightarrow 0$, Eq. (3) can be re-written as:

$$
W_{L}=\gamma_{L}(1+\cos \theta)=2\left(\gamma_{L}^{L W} \gamma_{S}^{L W}\right)^{1 / 2}+I_{S L}^{A B}
$$

in which we introduced the Lifshitz-van der Waals components of the surface energy of the liquid and solid, respectively, and gathered all other interactions between solid and liquid into the $I_{S L}^{A B}=0$ term [9]. The $\mathrm{LW}$ and $\mathrm{AB}$ interactions may be tuned by varying the nature of the liquid, which can be apolar like diodomethane or strongly polar like water. As a consequence, a plot of $\cos \theta$ as a function of the ratio $x=\sqrt{\gamma_{L}^{L W}} / \gamma_{L}$ will be linear if, and only if, $I_{S L}^{A B}=0$, which will be observed if the liquids are apolar, or the solid is apolar, or both. Furthermore, the slope of this plot will supply a measure of $2 \sqrt{\gamma_{S}^{L W}}$ whereas this plot goes to $\cos \theta=-1$ when $\mathrm{x} \rightarrow 0$ if, and only if, $I_{S L}^{A B}=0$.

A typical example of this plot is shown in Fig. 2. The five liquids that were used to produce the data are introduced in the next section. The samples for contact angle measurements (also described in the next section) were PTFE, a famous apolar solid with very reduced surface energy, a single crystal of pure alumina designed for optical windows, which is in contrast a very polar solid, and a single grain of an Al-Pd-Mn icosahedral quasicrystal grown by Czochralsky pulling from the liquid alloy [10]. This material is covered by a thin layer of alumina oxide in ambient air. Surprisingly, the plot of $\cos \theta$ versus $x$ is linear and resembles that of PTFE, which is apolar, yet with a larger slope. It is not comparable to that of the pure oxide, which departs from a linear plot and does not tend to $\cos \theta=-1$ for $x=0$. This clearly signs the polar nature of the $\mathrm{Al}_{2} \mathrm{O}_{3}$ surface. As a consequence, the surface energy of the quasicrystalline material must show a vanishingly small $\mathrm{AB}$ component, which is in strong contrast to the behaviour of the covering oxide and to what is known for the pure metal equipped with the same native oxide.

The motivation of our study was thus to better understand the origin of such a strange behaviour and to see if it can be traced to a specific property of the metallic substrate itself, thus taking our interpretation away from classical literature. To this end, we have 
studied a large number of Al-based compounds, crystalline as well as quasicrystalline, that can be grown out of Al-TM (TM: transition metal) alloy systems upon conventional metallurgical techniques since they are all stable. Using the very same samples, we have gained insight into their electronic structure, using soft X-ray spectroscopy. We have characterized their surface oxide layers by direct microscopy observations and XPS experiments. We have measured their roughness and assessed their wettability, using contact angle determinations, most often against water, but also against other types of liquids. Finally, we came to the conclusion that the reversible adhesion energy of water on those surfaces is essentially of Lifshitz-van der Waals type whenever the oxide layer is thin enough and the complexity of the metallic substrate large (or the unit cell of the compound contains at least few hundreds atoms). This result is in line with the selection of certain quasicrystalline alloys for the industrial production of low-stick cookware that was secured in patents by one of us long ago [11, 12].

Thus, the article is organised in four sections. The next one deals with experimental details, the third one with experimental results (effect of roughness and of the oxide layer, influence of the electron density of states underneath the oxide), the forth section is dedicated to a model that interprets the data, and the fifth raises few conclusions.

\section{Methods}

Samples in the shape of cylindrical pellets of $2 \mathrm{~cm}$ diameter and few millimetres thickness were prepared first by melting the raw constituents in a levitation induction furnace under argon protective atmosphere. The ingot thus obtained was then crushed to produce a powder with a mesh size in the range $20-50 \mu \mathrm{m}$, which was used later on for sintering under uniaxial pressure, also under protective atmosphere as explained elsewhere [13]. The stoichiometry of the sample was chosen in such a way as to produce a single phase material, which could be easily assessed by X-ray diffraction and optical metallography. Crystal structures considered in the present study varied from that of the pure metal (fcc $\mathrm{Al}$ and $\mathrm{Cu}$ ), pure oxide $\left(\mathrm{Al}_{2} \mathrm{O}_{3}\right)$ to that of a number of compounds, some having a rather simple unit cell with only few atoms like the $\omega-\mathrm{Al}_{7} \mathrm{Cu}_{2} \mathrm{Fe}$ compound with 48 atoms per unit cell (at/uc) or the $\gamma$-brass Al-Cr-Fe compound with 54 at/uc [14] to more complex ones like the $\beta$-AlCuFe CsCl-type of phase, which comprises only two crystallographic sites, but onto which the chemical species and vacancies order, thus forming superstructures with increasingly large unit cell [15]. Depending on composition, the reciprocal space of those compounds shows diffraction spots that depart from fivefold symmetry in varying extent, as illustrated in Fig. 3, which explains why they are often called approximants (of a quasicrystal) [16].

Quasicrystals from $\mathrm{Al}-\mathrm{Cu}-\mathrm{Fe}$ and $\mathrm{Al}-\mathrm{Pd}-\mathrm{Mn}$ systems [7, 8] were studied as well, including a single grain icosahedral quasicrystal of nominal composition $\mathrm{Al}_{72} \mathrm{Pd}_{20} \mathrm{Mn}_{8}$ (compositions are quoted either in number of atoms per unit formula, as given above for the $\omega$-compound, or in at. \% as is the case in this formula). The surface of these samples was prepared by mechanical polishing under a flow of water, avoiding especially using diamond paste in such a way that no lubricating or organic material could be trapped in voids opening at the surface. Several abrasive papers covered with corundum powder were used down to grit 4000 . The final roughness of the surface was characterized using a Dimension 3000 atomic force microscope (AFM) from Digital Instrument 

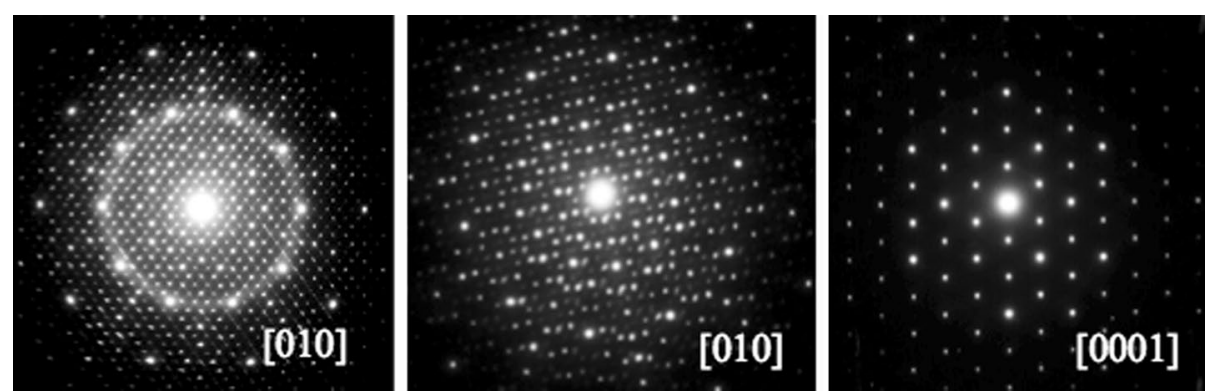

Fig. 3 The Al-Cr-Fe(-Cu) system is rich in approximant compounds, depending on the respective concentrations of the transition metals. The figure illustrates three different examples, using selected electron diffraction patterns taken along the pseudo fivefold direction of the crystal. The compound shown left contains several thousands at/uc, the one in the middle, few hundreds and the one right, only few tens. The complexity of such structures may be indexed according to a so-called complexity index, which is equal to the natural logarithm of the number of atoms per unit cell, or $\beta_{C}=\operatorname{Ln}$ (number of at/uc). The filling of reciprocal space, which is directly visualized in the figure, also reflects that complexity

used in tapping mode and expressed in terms of root mean square (RMS) values. The thickness of the native oxide on top of each sample was studied by transmission electron microscopy (TEM), using thin specimens cut perpendicular to the oxide layer (see below) and observed on a $200 \mathrm{kV}$ TEM from JEOL. We also obtained the value of the thickness from XPS measurements, based on Strohmeier's procedure [17]. For selected samples, we artificially monitored the thickness of the alumina layer by depositing a thin layer of aluminium atoms in ultra-high vacuum at liquid nitrogen temperature. This Allayer was then oxidised in situ at room temperature to form of supplementary layer of controlled thickness, which was assessed later on as explained above. Several successive such experiments allowed us to produce series of experiments, keeping the metallic substrate unchanged, but with oxide layers of different and controlled thicknesses.

Contact angles were measured on freshly polished surfaces using a sessile droplet device purchased from Digidrop ${ }^{\circledR}$. The volume of the droplet was fixed to $1 \mu \mathrm{l}$ by a syringe, the piston of which was equipped with a micrometric screw. We used five different liquids: (a) ultrapure water, (b) glycerol, (c) ethylene glycol, (d) diodomethane, and (e) tricresyl phosphate whose surface energies are listed in Table 1. Series of 10-15 measurements of the contact angle were obtained, using the three points determination software the device is equipped with, which supplied sufficient accuracy and reliability. Making use of the micrometric screw, we could also measure the advancing and receding contact angles of ultrapure water on selected specimens such as multigrained

Table 1 Characteristics of the five liquids used in the present study

\begin{tabular}{llll}
\hline Name of liquid & $\boldsymbol{\gamma}_{\boldsymbol{L}}$ & $\boldsymbol{\gamma}_{\boldsymbol{L}}^{\mathbf{L W}}$ & $\boldsymbol{Y}_{\boldsymbol{L}}^{\boldsymbol{A B}}$ \\
\hline Water & 72.8 & 21.8 & 51.0 \\
Glycerol & 64 & 34.0 & 61.3 \\
Ethylene glycol & 48 & 29.0 & 48.9 \\
Diodomethane & 50.8 & 50.8 & 0 \\
Tricresyl phosphate & 40.9 & 40.9 & 0 \\
\hline
\end{tabular}

Liquids listed in the left hand side column appear in the same order as they do along the x-axis in Fig. 2 
$\mathrm{Al}_{62} \mathrm{Cu}_{25} \mathrm{Fe}_{13}$ icosahedral quasicrystal (i-ACF hereafter) and $\mathrm{Al}_{72} \mathrm{Pd}_{20} \mathrm{Mn}_{8}$ single grain (i-APM hereafter). The effect of surface roughness was accessed, based on a series of contact angle measurements of water deposited on various samples of i-ACF that were polished down to different abrasive paper grades, thus varying their roughness.

Correlation to electronic structure data was based on measurements of partial density of $\mathrm{Al} 3 \mathrm{p}$ states at the Fermi energy [labelled $n\left(E_{F}\right)$ hereafter], which were supplied by soft $\mathrm{x}$-ray emission spectroscopy as explained in [18]. We used pure fcc aluminium as a reference material. Such a metal is a good example of a nearly-free electron system characterized by $n\left(E_{F}\right)=0.5$ if the maximum of the partial electronic density is set to 1 . Our samples are not free-electron systems anymore [16], which is characterized by the formation of a pseudo-gap at the Fermi energy [19] and therefore a decrease of $n\left(E_{F}\right)$ compared to the free-electron case (Fig. 4). We obtained a large number of values of $\mathrm{n}\left(\mathrm{E}_{\mathrm{F}}\right)$ for a broad series of crystalline compounds and quasicrystals, which was already published [18] and included some of the samples used for the present wetting experiments. Figure 5a shows a revised, so far unpublished, version of $n\left(E_{F}\right)$ plotted as a function of the average number of electrons per atom e/a computed for many compounds (not necessarily identical to those used for contact angle measurements in the present study). In this figure, the $\mathrm{x}$-axis represents the average number of electrons per atoms computed for each compound by referring to a recently published list of elemental values of e/a [20]. The same data is shown in Fig. 5b as a function of the complexity index [21], or the natural logarithm of the number of atoms present in each primitive unit cell. A discussion of electronic structure data may be found elsewhere [22].

\section{Results}

\section{Effect of roughness on the reversible adhesion energy of water}

In the following of this article, we shall substitute $\mathrm{H}_{2} \mathrm{O}$ for $\mathrm{L}$ to index the reversible adhesion energy of (ultrapure) water. We assessed the effect of roughness on this quantity by varying the polishing conditions of a sample made of sintered $\mathrm{Al}_{62} \mathrm{Cu}_{25} \mathrm{Fe}_{13}$ powder that

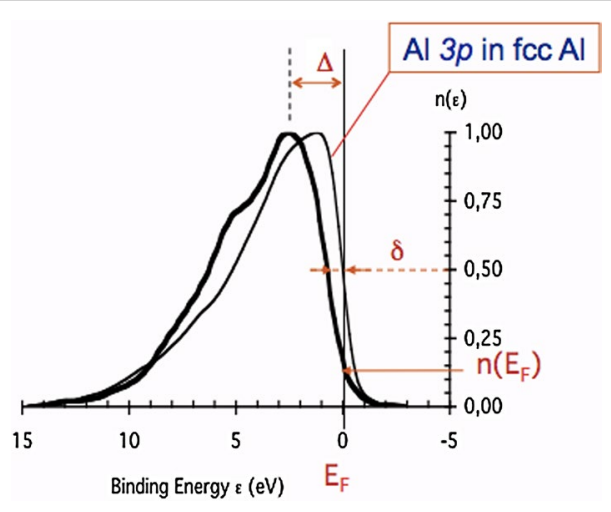

Fig. 4 Partial density of Al $3 p$ states (Al3p DOS) measured in fcc aluminium compared to the one found in the i-ACF quasicrystal. In this later compound, the Al3p DOS is shifted toward higher binding energies ( $\delta$ at half maximum, $\Delta$ at maximum) due to the formation of a pseudo-gap at the Fermi energy $\left(E_{F}\right)$ set to zero binding energy. As a consequence, the number $n\left(E_{F}\right)$ of Al3p states at $E_{F}$ is reduced compared to that in $f c c$ Al, for which $n\left(E_{F}\right)$ is by definition of the Fermi-Dirac distribution for a free electron system taken equal to 0.5 when the maximum of the DOS is normalized to 1 


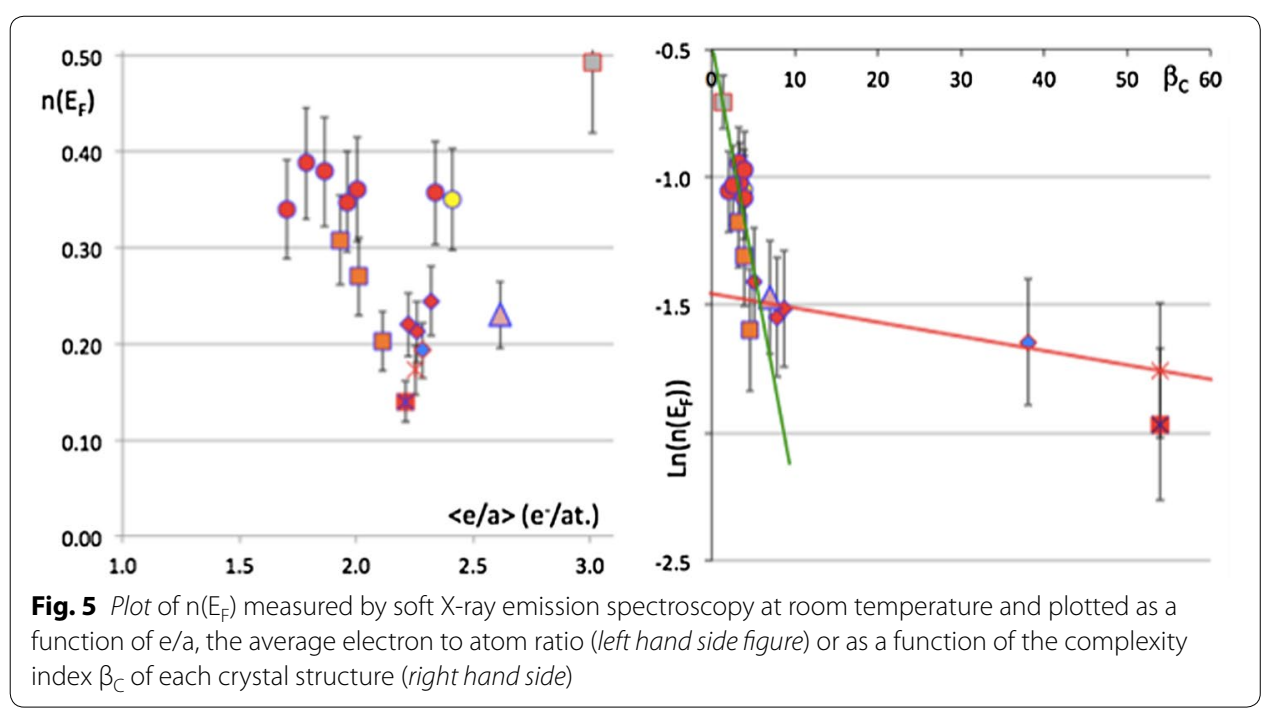

was annealed to eliminate any residual $\beta$-AlCuFe phase [15]. This phase is easily trapped in a metastable state while cooling the liquid alloy as a result of its formation through a peritectic reaction at $820^{\circ} \mathrm{C}$ [23]. Polishing was performed on corundum abrasive paper under water, using grades ranging from 800 down to 4000, thus decreasing the RMS roughness by about an order of magnitude. The resulting contact angles observed either parallel or perpendicular to the polishing direction are shown in Fig. 6. Both converge towards a same value, around $\theta=92^{\circ}$, up to experimental uncertainty.

Roughness plays an important role in wetting phenomena [24]. It is therefore important to make sure that the preparation of the surface minimizes, if not cancels, the effect of roughness artefacts, and is perfectly reproducible. This is the case with the procedure we have selected. Henceforth, we shall consider that the scatter of $\mathrm{W}_{\mathrm{H}_{2} \mathrm{O}}$ values does not result from surface roughness, because it is all the same after proper polishing down to 4000 grit paper for all relevant materials (CMAs) considered in the present study (Fig. 7).

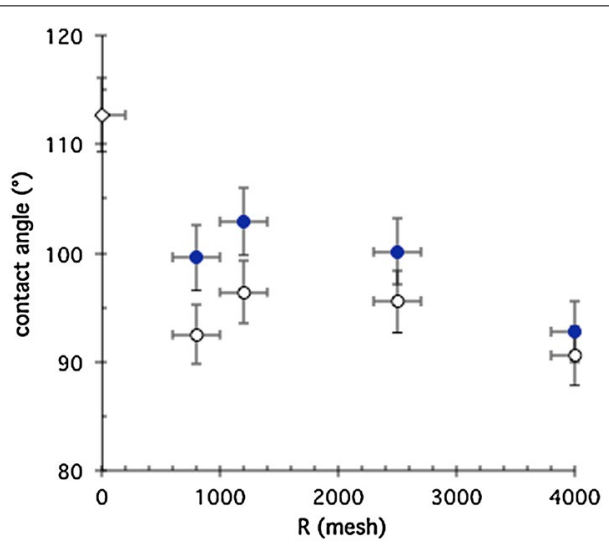

Fig. 6 Contact angle $\theta$ measured on a specimen of O1-AlCuFeCr approximant after polishing using corundum abrasive papers of increasing grit size. Open dots are for data obtained when viewing the droplet along the direction of polishing and solid dots for measurements perpendicular to it. The two sets of data converge to the same value at sufficiently low roughness, or high mesh size 


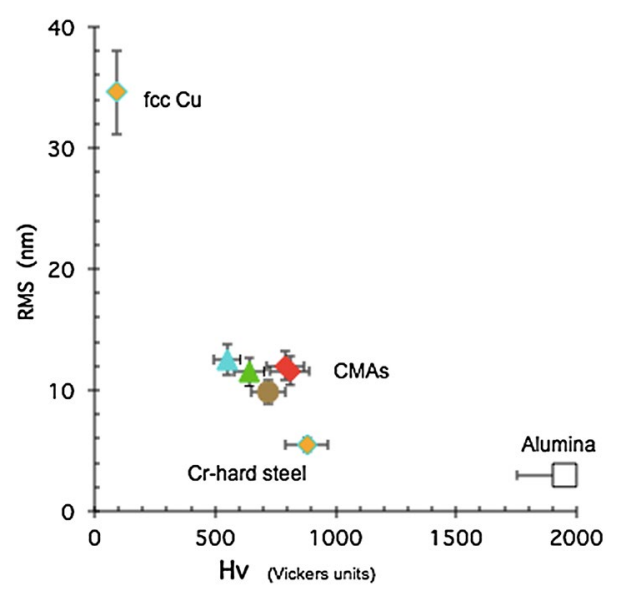

Fig. 7 RMS values observed on samples of varying Vickers hardness (load $0.5 \mathrm{~N}$ ) after the final polishing step described in text. The scatter of the data for complex metallic alloys (CMA) is negligible and implies that roughness does not influence the $\mathrm{W}_{\mathrm{H}_{2}} \mathrm{O}$ measured for those samples

Similarly, in such conditions, advancing and receding contact angles were checked to be the same within experimental accuracy.

\section{Effect of oxide layer thickness on $\mathrm{W}_{\mathrm{H}_{2} \mathrm{O}}$}

Oxidation of Al-based compounds is well known to take place at room temperature (RT), especially in water [25], and even more so at temperatures comprised between RT and the peritectic temperature [26]. We have therefore studied the contact angle of water with specimens that were heat treated in a way as to monitor the nature, thickness and roughness of the oxide layer grown in ambient air or oxidized in situ after depositing thin layers of pure aluminium on a $\gamma$-AlCrFe sample placed in a vacuum chamber.

Following the study by Bonhomme [27], let us start with the effect of oxidation significantly above room temperature. Three freshly polished samples of i-ACF, O1-AlCuFeCr approximant (see Fig. 3 left) and $\gamma$-AlCrFe crystal (see Fig. 3 right) were heat treated under ambient air at $450{ }^{\circ} \mathrm{C}$ for $10 \mathrm{~h}$, then abandoned in ambient atmosphere for $120 \mathrm{~h}$ and heat treated again at $200{ }^{\circ} \mathrm{C}$ for $2 \mathrm{~h}$ [27]. The time interval elapsed before, between and after the heat treatments is shown in Fig. 8. Contact angle measurements were performed with pure water, without any further polishing of the surface, at the times marked by numbers in the left side of the figure. Clearly, the contact angle moves up and down, from a value characteristic of low stick of water against that surface to a value specific of high wetting (or high $\mathrm{W}_{\mathrm{H}_{2} \mathrm{O}}$ ). The second, low contact angle $\theta$, is typical for naked alumina. It would not be different on oxidized aluminium nor is it on other types of Albased CMA that are submitted to high temperature annealing in air [28]. The first value is directly related to the contamination of the surface by organic molecules as a XPS scan easily teaches us. We insist here that the results reported in the coming sections of the present article, which form the heart of our paper, have no relationship whatsoever with this behaviour. We have measured the thickness of the alumina layer-around $35 \mathrm{~nm}$ and determined that it is amorphous (Fig. 9), in spite of the elevated heat treatment 

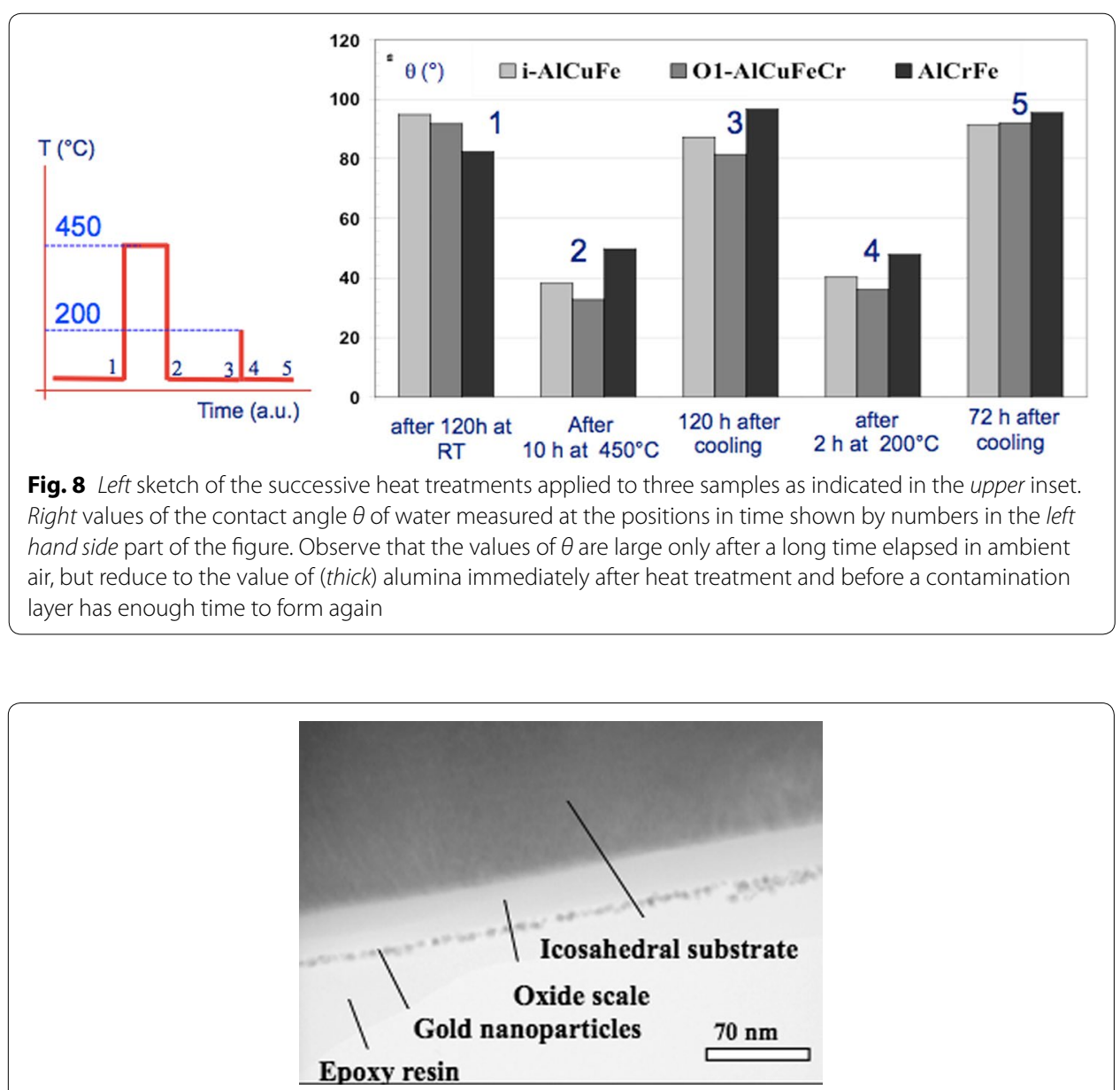

Fig. 9 Direct observation of the alumina layer grown on a i-ACF sample by heat treating at $500^{\circ} \mathrm{C}$ in air for $144 \mathrm{~h}$. The upper surface of the oxide is marked by gold nanoparticles before preparation of the thin foil, to make it easier to identify. The thickness is found around $35 \mathrm{~nm}$

temperature of $450^{\circ} \mathrm{C}$. Specimens we shall deal with in the following are equipped with a much thinner oxide layer, which changes the situation completely.

The situation is different because the value of the contact angle increases with decreasing thickness of the oxide, or conversely, the reversible adhesion energy of water is going down with increasing thickness of the oxide layer as long as it keeps under $10 \mathrm{~nm}$. We established such experimental evidence by evaporating $\mathrm{Al}$ atoms onto a sample of $\gamma$-AlCrFe kept at nitrogen temperature, then flowing pure oxygen at room temperature to the surface to grow in situ in the evaporator an alumina layer of controlled thickness. The choice of the process temperatures prevented any atomic transport from substrate to layer, so that this layer was made of pure, amorphous alumina. The values of $\mathrm{W}_{\mathrm{H}_{2} \mathrm{O}}$ measured on such samples immediately after growth of the oxide are shown in Fig. 10. They exhibit an experimental result that is counter-intuitive and cannot be predicted from published literature. We could also monitor the thickness of the native oxide layer grown on our Al-based samples in a restricted range between 2 and $10 \mathrm{~nm}$ and determine its value as explained in "Methods". We thus observed that the value of $\mathrm{W}_{\mathrm{H}_{2} \mathrm{O}}$ was systematically found lower for the thicker oxide layer (the size of the relevant symbols in 


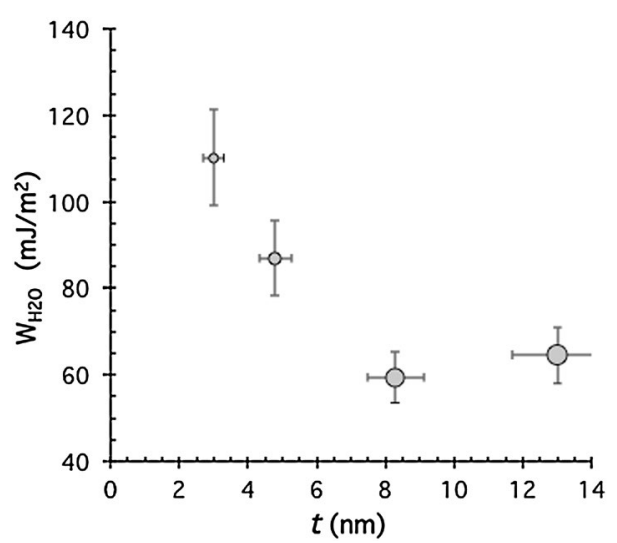

Fig. 10 Evolution of $\mathrm{W}_{\mathrm{H}_{2} \mathrm{O}}$ with increasing thickness $t$ of the oxide layer grown in situ on top of a sample of $\gamma$-AlCrFe approximant (see right side of Fig. 3). Observe the decrease of $\mathrm{W}_{\mathrm{H}_{2}} \mathrm{O}$ with $t$ increasing up to a value of about $10 \mathrm{~nm}$ where $\mathrm{W}_{\mathrm{H}_{2} \mathrm{O}}$ shows a marked minimum

Figs. 10 and 12 is in proportion to the oxide thickness). The general trend of $\mathrm{W}_{\mathrm{H}_{2} \mathrm{O}}$ versus thickness $t$ of the oxide is between linear and parabolic, as exemplified by the values shown in Fig. 10 up to $t=10 \mathrm{~nm}$.

\section{Lifshitz-van der waals and acid-base components of the surface energy}

The values of $\gamma_{S}^{L W}$ and $I_{S L}^{A B}$ (see "Methods") can be derived from contact angle measurements, using several liquids with varying LW and polar components as already explained in this paper. We did such measurements for a number of Al-based CMAs, including quasicrystals, as well as for a single grain of pure (crystalline) alumina and a sample of aluminium metal covered with its native (amorphous) oxide in ambient air. The results are summarized in Fig. 12. They are ordered along the x-axis according to the Al3p DOS measured at the Fermi energy, which is displayed in Fig. 3. The LW component is not very much sensitive to the sample nature and falls between the values characteristic of pure alumina (represented by an open square in the left hand side of Fig. 11) and that of fcc-Al (represented by a cross). In contrast, $I_{S L}^{A B}$ values are found well below the value corresponding to alumina, and to a lesser extent, that for fcc-Al. Some values are close to zero and correspond to the most complex compounds, which as Fig. 3 shows, also yield the lowest $\mathrm{n}\left(\mathrm{E}_{\mathrm{F}}\right)$. An approximately parabolic increase of $I_{S L}^{A B}$ is observed in the range above these data and up to the data for fcc-Al. This behaviour will be clarified in "Interpretation", but it cannot be assigned anymore to the classical concept of acid-base interactions, which would vary randomly in the vicinity of the value for pure $\mathrm{Al}_{2} \mathrm{O}_{3}$ since all specimens are covered by this oxide (the difference between the two data points for bulk alumina and oxidized fcc- $\mathrm{Al}$ is representative of the distinct crystal structures of these materials, one being single crystalline and the other amorphous). We will come back to the actual meaning of the $I_{S L}^{A B}$ term in "Interpretation" below.

\section{Correlation between $\mathrm{W}_{\mathrm{H}_{2} \mathrm{O}}$ and $\mathrm{n}\left(\mathrm{E}_{\mathrm{F}}\right)$}

Our strangest result is presented in Fig. 12. We observe a direct correlation between $\mathrm{W}_{\mathrm{H}_{2} \mathrm{O}}$ for many CMA samples and the values of $n^{2}\left(E_{F}\right) / t^{2}$. The choice of this specific 

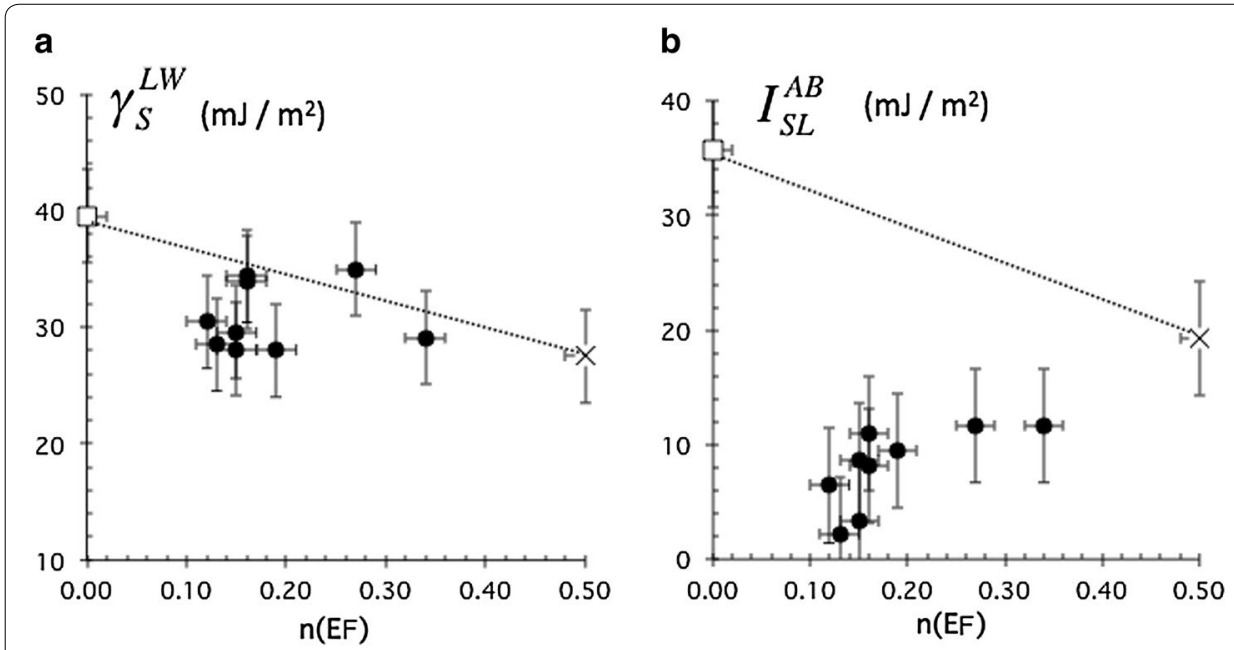

Fig. 11 Lifshitz-van der Waals and $A B$ components of the surface energy deduced from contact angle measurements using several liquids deposited on the surface of Al-based compounds. The open square is for single crystalline alumina and the cross for aluminium metal equipped with its native oxide. The dotted line guides the eye between the data for alumina and for fcc-Al. Observe that all Lifshitz-van der Waals data points fall in a range below the value for bulk alumina and close to that of the pure, oxidized metal whereas all values of the $A B$ component are clearly below that of the (oxidized) metal, and far below that for alumina. The specimens that exhibit a vanishingly small $A B$ component are the most complex ones and include the quasicrystalline i-ACF and i-APM samples

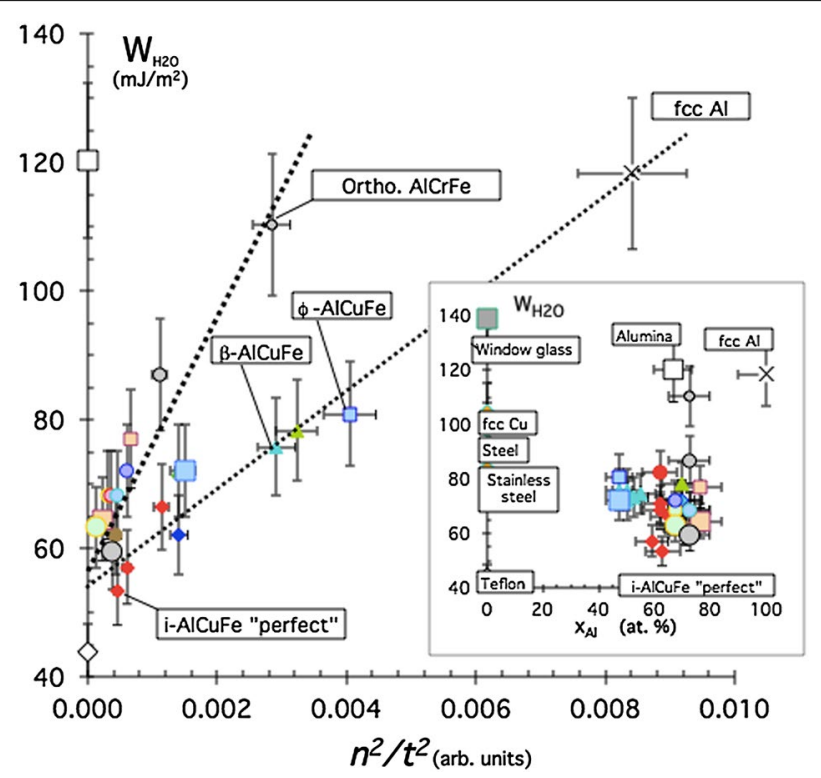

Fig. 12 Plot of the value of the reversible adhesion energy of water on the surface of a large variety of samples as a function of the $n^{2}\left(E_{F}\right) / t^{2}$ ratio. The partial Al3p DOS, $n\left(E_{F}\right)$ is reported in Fig. 3 for the very same specimens. Values of the oxide layer thickness, $t$, were deduced from XPS experiments as explained in "Methods". For the sake of comparison, we also give $\mathrm{W}_{\mathrm{H}_{2} \mathrm{O}}$ data, but for materials that contain no Al3p states (e.g. window glass or stainless steel) and therefore place the data at the origin of the $x$-axis. The inset shows the same information, but plotted as a function of the Al-concentration in the specimens. When relevant, we used the same symbol for a sample that we could obtain with different values of the oxide thickness (the higher the thickness, the larger the size of the symbol). The data align along two sets of lines, depending on whether they contain a large concentration of $3 \mathrm{~d}$ metals ( $\mathrm{Cr}, \mathrm{Mn}$, Fe, upper line) or merely Cu (lower line) 
correlation will be explained in the next section. At this stage, it is important to stress that the DOS at the Fermi energy experienced by soft X-ray emission spectroscopy is a bulk property, which is probed several tens of nanometres below the extreme surface of the material, where according to literature interactions that determine the equilibrium shape of a liquid are supposed to take place. The reason why Fig. 12 exhibits two distinct branches comes from the fact that we had two series of samples, one containing essentially $\mathrm{Al}, \mathrm{Cu}$ and little $\mathrm{Fe}$, the other $\mathrm{Al}, \mathrm{Cr}$ and other transition metals like $\mathrm{Mn}, \mathrm{Fe}$ or Ni. The electronic density of states of the first series is characterized by a small density of $d$ states at the Fermi energy arising from the transition metals, whereas the other shows a large density of $d$ states at $E_{F}$ [29]. Since soft X-ray emission gives access only to partial densities (here, the Al3p DOS), we cannot use the total DOS, which would be relevant in Fig. 12. Therefore, the data split in two branches, depending on the presence or not of a high density of $d$-states at $E_{F}$, but this does not change our conclusion regarding the coupling experimentally observed between a bulk property $\left[n\left(E_{F}\right)\right]$ and the equilibrium shape of a droplet of water. Furthermore, the two branches converge towards a same value of $\mathrm{W}_{\mathrm{H} 2 \mathrm{O}}$, which is the one expected if only $\mathrm{LW}$ interactions would define the surface energy of the solid whereas $I_{S L}^{A B}$ would be 0 .

At this stage, let us summarize our findings:

(a) Lifshitz-van der Waals interactions are originating in the layer of $\mathrm{Al}_{2} \mathrm{O}_{3}$ oxide that is always present at the surface of our samples in ambient air (and in water on the other side of the interface). They are not different in nature from the ones present on aluminium metal placed in the same conditions.

(b)Other surface interactions are essentially different from the ones usually assigned to acid-base interactions. They originate from the bulk of the material and correlate to the density of electronic states present in the material under the oxide layer. We will call those states the "Fermi sea" of energy $\mathrm{E}_{\mathrm{F}}$. Furthermore, the oxide layer plays a role, although it acts as an inert spacer, in proportion to its thickness $t$ (or to $t^{2}$ ).

With these findings, we are brought pretty far from standard literature. Therefore, we will base our interpretation of the experimental results on a model that considers the interaction of the water dipoles with the images they form in the Fermi sea.

\section{Interpretation}

Let us first consider one single dipole located in the close vicinity of the triple line that marks the border of the droplet on the solid surface (Fig. 13). This dipole is at a distance $\mathrm{d}$ from interface with the top of the oxide layer (taken as the origin of coordinates along the $\mathrm{z}$ direction). According to image dipoles theory [30], the image that forms from this dipole will be found at a distance $\mathrm{d}^{\prime}=\mathrm{d}+t / \mathrm{\kappa}$ where $\mathrm{\kappa}=\varepsilon_{1} / \varepsilon_{2}$ is the ratio between dielectric permittivity of the oxide and water, respectively. Typical values of $\kappa$ fall in the range $0.1<\kappa<0.2$. As a consequence, the image dipole forms far below the lower side of the oxide barrier, which acts as an inert spacer, but repels images well inside the bulk of the compound, consistently with our observations above. The electrostatic interaction between the dipole and its image develop a force along the z-direction. As illustrated in Fig. 1, this force may modify the equilibrium of the surface tensions defined 


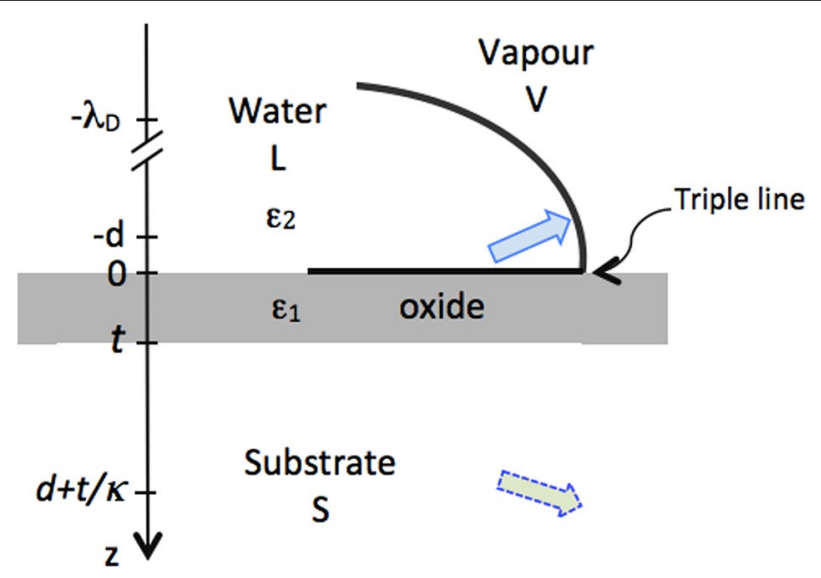

Fig. 13 Schematic representation of the triple line region, at the limit between vapour, liquid and solid substrate. Remarkable distances along the z-axis are defined in text. Only one water dipole and its image is represented

in that figure, and therefore the contact angle. We may evaluate this force, since it is proportional to the electric field created by the assembly of dipoles found within a layer of water of thickness equal to the Debye length $\lambda_{\mathrm{D}}$ on the one hand, and their image dipoles, on the other. We assume then that each constantly moving water dipole acts as a plane wave emitter. This wave is partly reflected and partly transmitted at every interface met underneath the liquid: the water-oxide $(\mathrm{w}-\mathrm{o})$ interface, and the oxide-substrate (o-s) interface (Fig. 14). At this later interface especially, transmission of the wave to the Fermi sea reservoir, the material itself, will be determined by its reflectivity coefficient $R$ in the infra-red range. This number approaches close to 1 for a good metal like fcc-Al, but is found significantly below unity for Al-based CMAs [31]. As a matter of facts, it is as low as $\mathrm{R}=0.6$ for a quasicrystal like $\mathrm{i}$-ACF. Again, this point is consistent with our findings: metallic aluminium will behave like pure alumina because the wave emitted by the water dipole is almost entirely confined within the oxide layer $(R \approx 1)$, whereas CMAs will show a different behaviour since absorption of the wave will be considerable $(\mathrm{R} \ll 1)$. Furthermore, such absorption will ensure the coupling with the Fermi sea and establish both the dependence upon $\mathrm{n}\left(\mathrm{E}_{\mathrm{F}}\right)$ and $t$.

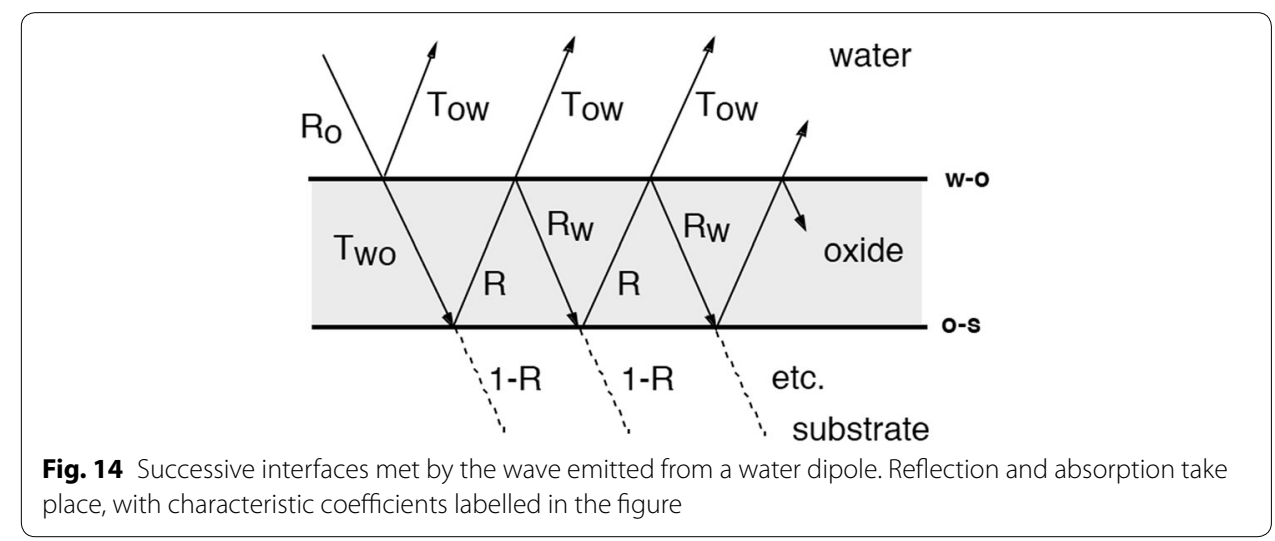


Detailed analytical computation of the effect may be found elsewhere [32]. We need just in the present context to give the main steps and come quickly to the final result. Dipole-dipole interactions establish an electric field that varies as $r^{-6}$, where $r$ is the separation distance. Integrating over all possible movements of the dipoles located within the $0<\mathrm{d} \leq \lambda_{\mathrm{D}}$ range of distances and summing up all dipole contributions leads to an electric field at distance $\mathrm{d}=0$ that is dominated by a term proportional to $t^{-2}$, in full agreement with the results shown in Fig. 12.

The force that applies on the triple line at the origin of coordinates (Fig. 1) is then proportional to the electric field created at the same position by the dipole-image dipole interactions. This field, to first approximation, is given by:

$\mathrm{E}(0)=\frac{1}{16 \pi \varepsilon_{0}} \frac{1}{\mathrm{t}^{2}}\left[\kappa(1+\mathrm{R})^{2}\left(\kappa(1+\mathrm{R})^{2}-2\left(1-\mathrm{R}^{2}\right)\right)-\kappa^{-1}(1-\mathrm{R})^{2}\left(\kappa^{-1}(1-\mathrm{R})^{2}-2\left(1-\mathrm{R}^{2}\right)\right)\right] / \mathrm{R}^{2}$

To go a step further, we need to know $\mathrm{R}$ for each of the compounds of interest, which is not feasible yet. We shall therefore use an antsatz, which relates $R$ to $n\left(E_{F}\right)$ :

$$
R=\frac{1+2 n\left(E_{F}\right)}{2}
$$

that indeed fits the fragmented knowledge we have of the reflectivity index: $R=1$ in fcc $\mathrm{Al}$, when $\mathrm{n}\left(\mathrm{E}_{\mathrm{F}}\right)=0.5$ and $\mathrm{R}=0.6$ as for $\mathrm{i}-\mathrm{ACF}$ and $\mathrm{i}-\mathrm{APM}$, for which it is known that $n\left(E_{F}\right)=0.12$ (Fig. 5). The force that is proportional to $E(0)$, Eq. 5, is plotted in Fig. 15 (in arbitrary units) as a function of the ratio $\left(\mathrm{n}\left(\mathrm{E}_{\mathrm{F}}\right) / t\right)^{2}$ at unit thickness $(t=1)$ and for 3 different, but representative values of $\kappa$. Worth noticing, it shows a linear variation within the range of $n\left(E_{F}\right)$ values that are available yet, $0.12 \leq n\left(E_{F}\right) \leq 0.5$ (Fig. 5).

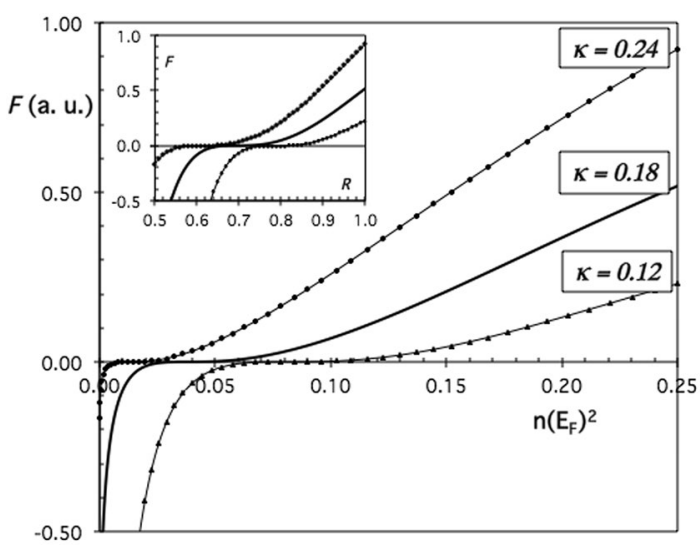

Fig. 15 Computation for $t=1$ of the force (in arbitrary units) that is proportional to the electric field created at position $z=0$ by the water dipoles and their images in the vicinity of the triple line. The computation is given for three different $\mathrm{k}$ ratios, which fall close to the conditions experienced by water deposited on an alumina layer. Observe the linear variation as a function of $n^{2}\left(E_{F}\right)$ within the range $0.1 \leq n^{2}\left(E_{F}\right) \leq 0.5$ of data that is available for the moment. The inset shows the same data, but with the $x$-axis labelled according to $R$ values 


\section{Conclusion}

Using wetting experiments and contact angle measurements, we have systematically investigated the surface energy of aluminium-based compounds equipped with their native oxide superficial layer. We have observed that, if the traditional Lifshitz-van der Waals interactions take their root in the oxide layer within the extreme surface layers of the specimens, this cannot be the case any longer for electronic and ionic interactions whenever oxide layers are thin enough $(t \leq 10 \mathrm{~nm})$. In this later case, we have found that such interactions, if they exist, are hidden by a much more important term that arises from the electronic cloud at Fermi energy that is located beneath the oxide layer, in the bulk of the alloy. We have estimated the resulting force that participate to the equilibrium shape of the liquid droplet and we have found that it varies in proportion to the square of the ratio between density of states at the Fermi energy divided by oxide layer thickness, in agreement with our experimental data.

We have observed that quasicrystals with high structural quality exhibit negligible electron/ionic exchange through the interface with the liquid, and that therefore, the sole contribution to the surface energy arises from Lifshitz-van der Waals interactions, which is quite new for substrates made of metals. This property is easy to achieve on an industrial scale. Therefore, it could be exploited to manufacture products that show reduced sticking to liquid or organic materials. The condition of high structural quality needs however to be strictly obeyed, for instance by annealing the device in order to eliminate any metastable phase inherited from high temperature processing [33]. Without such precaution, coatings, although of the appropriate composition, result in an awful mixture of undesired phases and show furthermore considerable concentration gradients that promote corrosion [34]. On top of this, the left hand side of Fig. 15 suggests that hydrophobic conditions may exist at very low values of $\left[n\left(\mathrm{E}_{\mathrm{F}}\right) / t\right]^{2}$ and for an appropriately selected value of $k$. Although we did not find yet a proper combination of compound with very low DOS at the Fermi energy, and oxide with very low dielectric permittivity, it is tempting to think that quasicrystalline coating come to the point when they could be considered attractive for the replacement of fluorine-containing surface layers [35].

Authors' contributions

JMD prepared the manuscript, based on his own contact angle measurements and data collected from literature. EBF, who did the soft X-ray emission spectroscopy measurements, revised the draft manuscript. All authors read and approved the final manuscript.

\section{Author details}

${ }^{1}$ Institut Jean Lamour (CNRS-Université de Lorraine UMR 7198), Parc de Saurupt, CS54840, 54011 Nancy Cedex, France. 2 LCPMR (Sorbonnes Université, Université Pierre et Marie Curie, UMR 7614), 11 rue P. et M. Curie, 75231 Paris Cedex 5, France.

\section{Acknowledgements}

The financial support of CNPQ and the hospitality offered by the Federal University of Paraiba, Brazil during the leave of absence of one of us (JMD) are gratefully acknowledged.

\section{Competing interests}

The authors declare that they have no competing interests. 


\section{References}

1. Young T. An essay on the cohesion of fluids. PhiL Trans Roy Soc. 1805;95:65.

2. Israelachvili J. Intermolecular and surface forces. London: Academic; 1985.

3. de Gennes PG. Wetting: statics and dynamics. Rev Mod Phys. 1985;57:827.

4. Tyson WR. Surface energies of solid metals. Can Metall Q. 1975;14:307.

5. Carré A. Phénomènes d'Interface. In: Briant J, editor. Paris: Technip. 1989.

6. Shechtman D, Blech, Gratias D, Cahn JW. Metallic phase with long-range orientational order and no translationnal symmetry. Phys Rev Lett. 1984;53(20):1951.

7. Tsai AP, Inoue A, Masumoto T. A stable quasicrystal in Al-Cu-Fe system. Jpn J Appl Phys. 1987;26:1505.

8. Tsai AP, Inoue A, Masumoto T. Stable decagonal Al-Co-Ni and Al-Co-Cu quasicrystals. Mater Trans JIM. 1989;30:463.

9. Good RJ. Contact angle, wetting, and adhesion: a critical review. J Adhes Sci Technol. 1992;6:1269.

10. Feuerbacher M, Thomas C, Urban K. Single quasicrystal growth. Quasicrystals. Weinheim: VCH Wiley; 2003. p. 2-25.

11. Dubois JM and Weinland P. Matériaux de revêtement pour alliages métalliques et métaux. French patent specification $n^{\circ} 18810559,1988$ \& US Patent Specification n5204191. 1993.

12. Dubois JM. So useful, those quasicrystals. Isr J Chem. 2011;51:1168

13. Dubois JM, Brunet P, Belin-Ferré E. Potential applications of quasicrystalline materials. In: Belin-Ferré E, Berger $C$, Quiquandon M, Sadoc A, editors. Quasicrystals, current topics. Singapore: World Scientific; 2000. p. 498-532.

14. Demange V, Anderegg JW, Ghanbaja J, Machizaud F, Sordelet DJ, Besser M, Thiel PA, Dubois JM. Surface oxidation of Al-Cr-Fe alloys characterized by X-ray photoelectron spectroscopy. Appl Surf Sci. 2001;173:327-38.

15. Dong C, Wang DH, Ge F. He FZ. In: Goldman Al, Sordelet DJ, Thiel PA, Dubois JM, editors. New horizons in quasicrystals. Singapore: World Scientific; 1997. p. 17-25.

16. Dubois JM. Useful quasicrystals. Singapore: World Scientific; 2005.

17. Strohmeier BR. An ESCA method for determining the oxide thickness on aluminum alloys. Surf Interf Anal. 1990;15:51.

18. Belin-Ferré E, Fournée V, Dubois JM. Al 3p occupied states in Al-Cu-fe intermetallics and enhanced stability of the icosahedral quasicrystal. J Phys Condens Matter. 2000;12(37):8159.

19. Traverse A, Dumoulin L, Belin E, Senemaud C. Electronic distributions in AlMn alloys. First results. In: Janot C, Dubois JM, editors. Quasicrystalline materials. Singapore: World Scientific; 1988. p. 399-403.

20. Mizutani U, Sato H, Inukai M, Nishino Y, Zijlstra ES. Electrons per atom ratio determination and hume-rothery electron concentration rule for P-based polar compounds studied by FLAPW-Fourier calculations. Inorg Chem. 2015;54:930-46

21. Dubois JM. Properties- and applications of quasicrystals and complex metallic alloys. Chem Soc Rev. 2012:41:6760-77.

22. Dubois JM, Belin-Ferré E. Electronic properties and complexity of Al-based complex metallic alloys. Acta Phys Pol, A. 2014;126:453-5.

23. Dong C, Dubois JM, de Boissieu M, Janot C. Neutron diffraction study of the peritectic growth of the $\mathrm{Al}_{65} \mathrm{Cu}_{20} \mathrm{Fe}_{15}$ icosahedral quasi-crystal. J Phys Cond Matter. 1990;2:6339-60.

24. Rivier N, Wetting on quasicrystals. In: Goldman Al, Sordelet DJ, Thiel PA, Dubois JM Editors. New horizons in quasicrystals: research and applications, Goldman A, Sordelt DJ, Thiel PA and Dubois JM, Editors. Singapore: World Scientific; 1997. p. 188-193.

25. Jenks CJ, Pinhero PJ, Chang SL, Anderegg JW, Besser MF, Sordelet DJ, Thiel PA. Surface oxidation of Al-Pd-Mn and AlCu-Fe alloys. In: Goldman A, Sordelt DJ, Thiel PA, Dubois JM, editors. New horizons in quasicrystals. Singapore: World Scientific; 1997. p. 157-64.

26. Weisbecker P, Bonhomme G, Bott G, Dubois JM. The oxidation at $500^{\circ} \mathrm{C}$ of AlCuFe quasicrystalline powders: a X-ray diffraction study. J Non-Cryst Sol. 2005;351:1630-8.

27. Bonhomme G, Oxidation of quasicrystals for industrial applications, PhD Thesis, Vandoeuvre les Nancy. http://www. sudoc.fr/171563794 (2003).

28. Bonhomme G, LeMieux M, Weisbecker P, Tsukruk VV, Dubois JM. Oxidation kinetics of an AlCuFeCr approximant compound: an ellipsometric study. J Non-Cryst Sol. 2004;334-335:532-9.

29. Belin-Ferré E, Dankhazi Z, Fontaine MF, de Weerd MC, Dubois JM. Electronic densities of states and pseudo-gaps in Al-based complex intermetallics. Croat Chem Acta. 2010;83(1):55-8.

30. Jennings PJ, Jones RO. Beyond the method of images - the interaction of charged particles with real surfaces. Adv Phys. 1988;37-3:341-58.

31. Demange V, Milandri A, de Weerd MC, Machizaud F, Jeandel G, Dubois JM. Optical conductivity of Al-Cr-Fe approximant compounds. Phys Rev B. 2002;65:144205-1-11

32. Dubois JM, Fournée V, Thiel PA, Belin-Ferré E. Measurements of contact angles of water on Al-based intermetallic surfaces. J Phys Cond Matter. 2008;20:314011 (10 pp)

33. Dubois JM. So useful, those quasicrystals. Isr J Chem. 2011;51:1168-75.

34. Sordelet DJ, Widener SD, Tang Y, Besser MF. Characterization of a commercially produced Al-Cu-Fe-Cr quasicrystalline coating. Mater Sci Eng. 2000;294-296:834-7.

35. Fehrenbacher L. Sputtering technique forms versatile quasicrystalline coatings. MRS Bull. 2011;36:581. 